\title{
An Oligomeric Ruthenium Polypyridyl Dye for Improved Stability of Aqueous Photoelectrochemical Cells
}

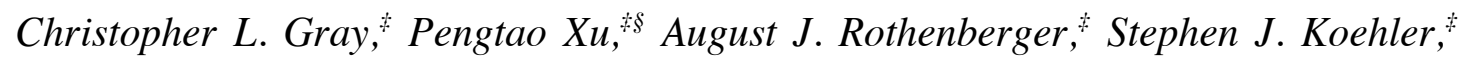 \\ Elizabeth Elacqua, ${ }^{\ddagger}$ Bratoljub H. Milosavljevic, ${ }^{+}$and Thomas E. Mallouk ${ }^{\ddagger *}$
}

${ }^{\ddagger}$ Department of Chemistry, The Pennsylvania State University, University Park, PA 16802, USA

'Department of Chemistry, University of Pennsylvania, Philadelphia, PA 19104, USA

\section{SUPPORTING INFORMATION (5 pp.)}

\section{Intensity-modulated photovoltage spectroscopy (IMVS)}

IMVS was conducted using an Autolab potentiostat (PGSTAT128N) in combination with an Autolab LED Driver. A $470 \mathrm{~nm}$ LED light (LDC470, Metrohm), driven by the LED Driver, was used as the light source. The electrolyte was either $10 \mathrm{mM}$ acetic acid/sodium acetate solution

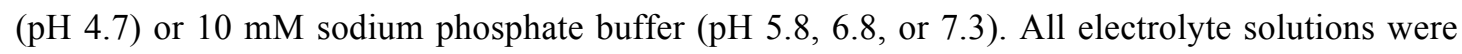
degassed by purging with Ar. IMVS measurements were carried out at ambient temperature $\left(23-24{ }^{\circ} \mathrm{C}\right)$ in the three-electrode configuration using $\mathrm{Ag} / \mathrm{AgCl}(3 \mathrm{M} \mathrm{NaCl})$ as the reference electrode and $\mathrm{Pt}$ wire as the counter electrode. All reagents were purchased from Sigma Aldrich and used without further purification. All potentials reported here are referenced to the reference electrode unless otherwise noted. In the IMVS experiments, the electrode was held at open-circuit potential by setting its current zero. The incident light was modulated in a sinusoidal fashion at a given frequency with a modulation magnitude of $10 \%$ of the DC level. Simultaneously, the electrode potential modulation at the same frequency was analyzed by the potentiostat. By scanning a range of frequencies, one can generate a Nyquist plot representing the real and imaginary parts of the frequency responses. Light intensity was measured by a Si photodiode (Thorlabs, S130C) and ranged from approximately $0.5-7.0 \mathrm{~mW} / \mathrm{cm}^{2}$.

In a photoelectrode, all the photo-injected electrons must recombine under open-circuit conditions. With the simplifying assumption of a first-order charge recombination process and a rate constant 
of $k_{1}$, the balance of charge injection and recombination can be expressed by equation (1):

$$
\frac{d n}{d t}=q A I-q k_{1} n(1)
$$

where $q$ is the electron charge, $A$ is the fraction of photons that result in electron injection, $I_{0}$ is the incident photon flux at steady state, and $n$ is the injected electron density.

Under constant illumination at an intensity of $\mathrm{I}_{0}$, the electron concentration reaches steady state and equation (1) can be written as follows:

$$
\frac{d n}{d t}=0=q A I_{0}-q k_{1} n_{0}
$$

Where $n_{0}$ is the injected electron density in $\mathrm{TiO}_{2}$ at steady state.

When the light is modulated with a frequency of $\omega$ and an amplitude of $M$, as represented in equation (3):

$$
I=I_{0}\left(1+M e^{i \omega t}\right)
$$

The response of $n$ at this frequency can be expressed as:

$$
n=n_{0}\left(1+m e^{i \omega t}\right)
$$

Where $\mathrm{m}$ is the magnitude of electron density modulation.

Using equations (1)-(4), we can solve analytically for $\mathrm{m}$ as follows:

$m=\frac{M}{1+\frac{i \omega}{k_{1}}}=\frac{M k_{1}^{2}}{k_{1}^{2}+\omega^{2}}-i \frac{M k_{1} \omega}{k_{1}^{2}+\omega^{2}}$

This expression for $\mathrm{m}$ suggests that when the scanned frequency $\omega$ equals $k_{1}$, the imaginary part of $m$ reaches a maximum. With a small light perturbation, we assume that the capacitance of the electrode is constant, and thus the modulation of $n$ translates to the frequency response of electrode potential that can be measured.

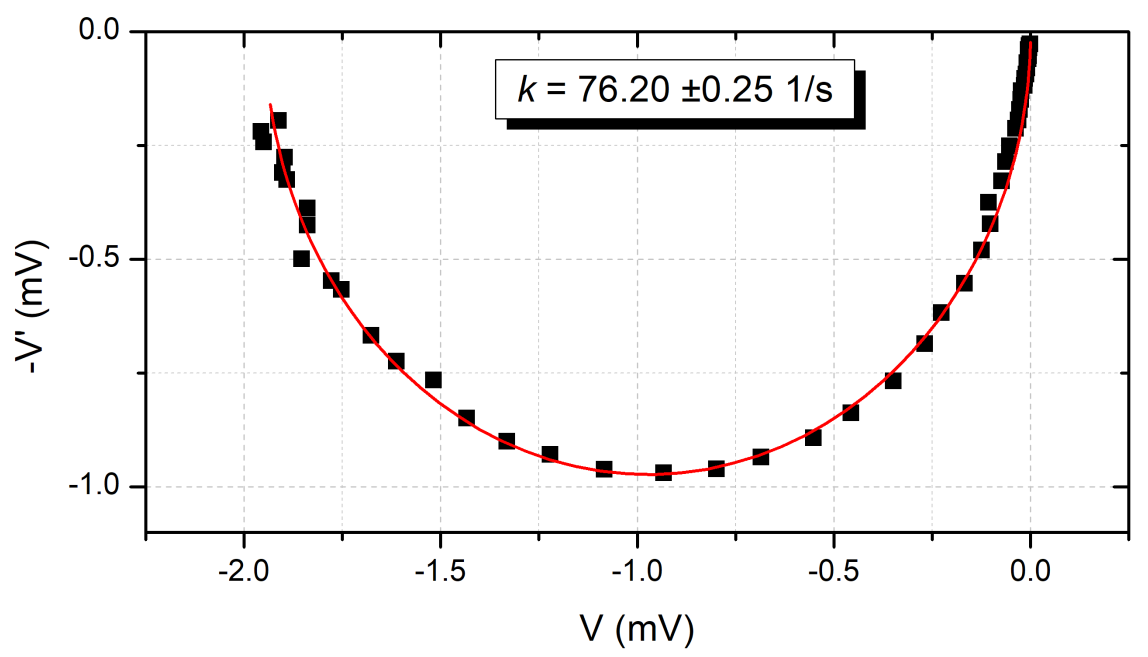

Figure S1. IMVS Nyquist plots of the measured sample (black) and the fitted line using equation (5) for a monomeric sample at a $\mathrm{pH}$ of 4.8 and a light intensity of $9.3 \mathrm{~mW} / \mathrm{cm}^{2}$. The fitted recombination rate $k$ is also shown. 

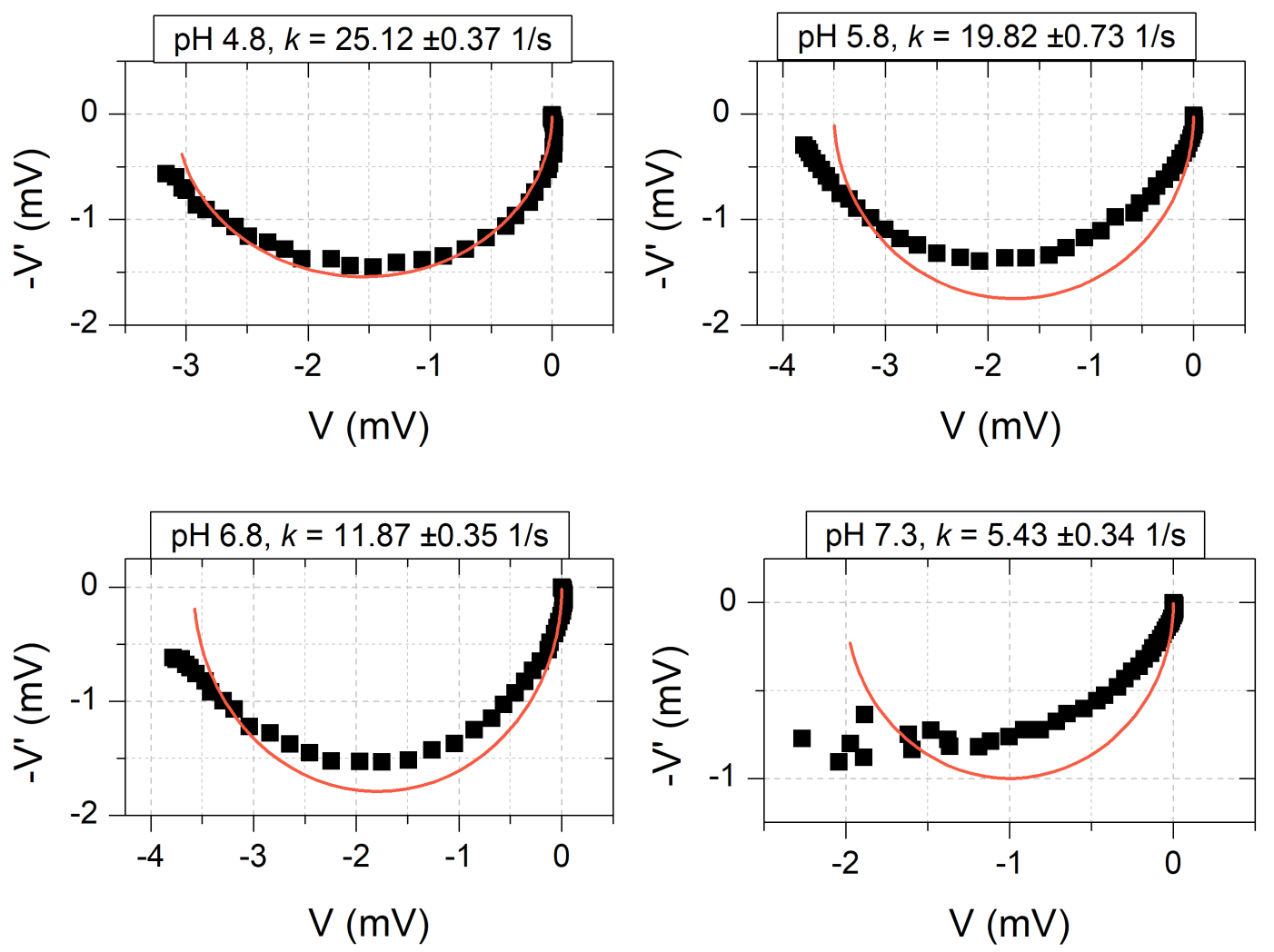

Figure S2. IMVS Nyquist plots of the measured sample (black) and the fitted line using equation (5) for a oligomeric samples at various pHs. The fitted recombination rate $k$ is also shown.

\section{MALDI-TOF Mass Spectrometry}

Samples were run using DHB (2,5-dihydroxybenzoic acid), dithranol, or trans-2-[3-(4-tert-butylphenyl)-2-methyl-2-propenylidene]malononitrile (DCTB) as the matrix. The oligomer suffered the least amount of fragmentation using DCTB and the results are shown in the figure below. 


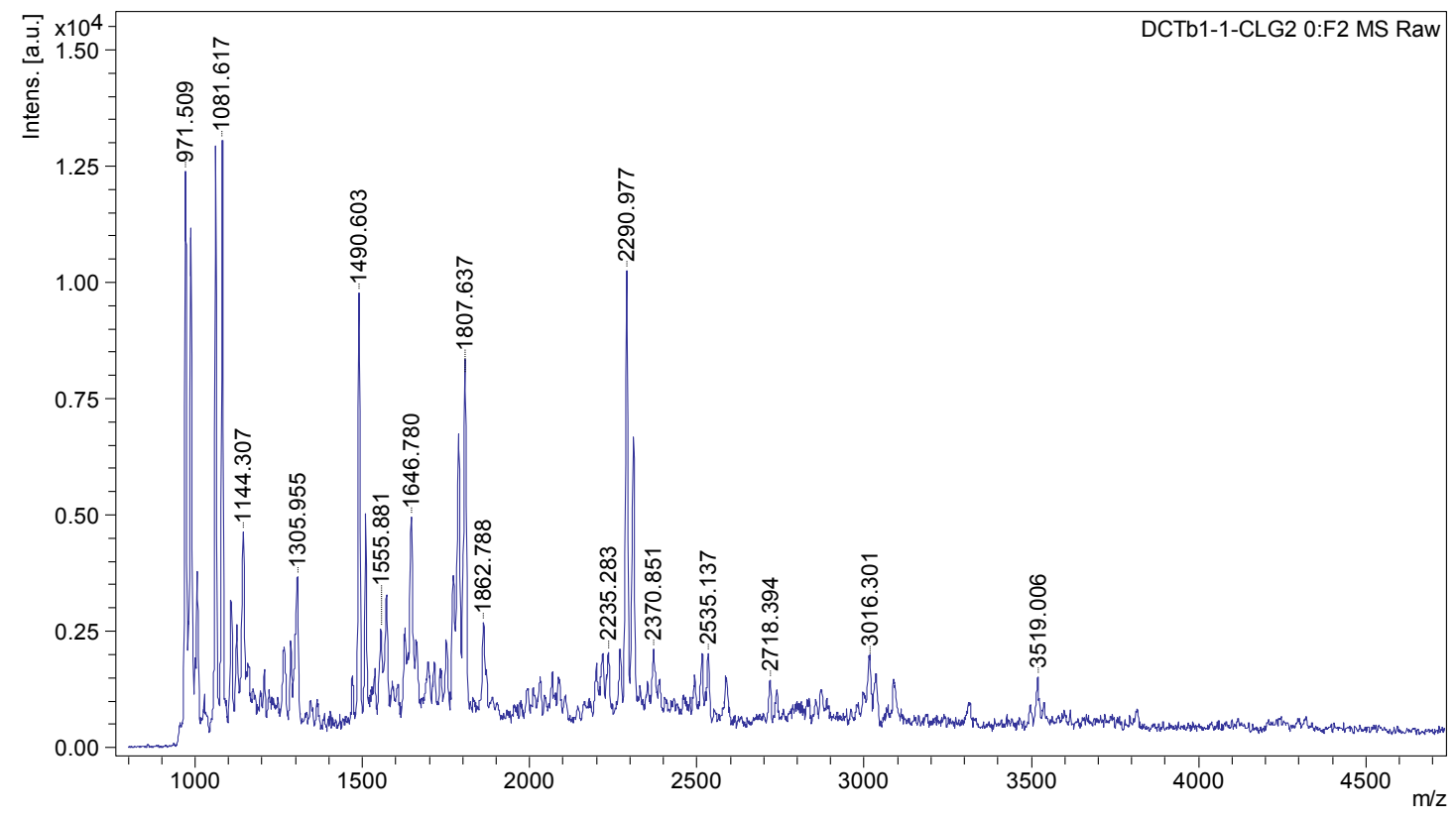

Figure S3. MALDI-TOF Mass spectrum of oligomer

\section{Pore Penetration by the Oligomeric Dye}

Samples were soaked in $2 \mathrm{~mL}$ solutions of $100 \mu \mathrm{M}$ of oligomer dye and then the $2 \mathrm{~mL}$ of $100 \mu \mathrm{M}$ monomer dye. Soaking in the monomer solution following the oligomer adsorption step resulted in an average relative decrease in absorbance of $1.5 \%$ across four samples. This result demonstrates that after the first oligomer adsorption step there is little unsensitized area of the electrode available for the smaller monomer to adsorb. In theory, if the oligomer were not adsorbing into all of the pores, then the second monomer adsorption step would dramatically increase the absorbance of the electrode. Samples 1 and 2 were made with $\mathrm{TiO}_{2}$ layers from one piece of scotch tape and the samples 3 and 4 were made with 2 layers of scotch tape

Table S1. Absorbances of samples from sequential oligomer and monomer adsorption

\begin{tabular}{|c|c|c|c|c|}
\hline & $\begin{array}{l}\text { Electrode Absorbance } \\
\text { after Oligomer } \\
\text { Adsorption (OD) }\end{array}$ & $\begin{array}{l}\text { Electrode } \\
\text { Absorbance after } \\
\text { Monomer } \\
\text { Adsorption (OD) }\end{array}$ & $\begin{array}{l}\text { Absolute } \\
\text { Difference (OD) }\end{array}$ & $\begin{array}{l}\text { Relative } \\
\text { Difference (\%) }\end{array}$ \\
\hline Sample 1 & 0.742 & 0.776 & 0.034 & 4.6 \\
\hline Sample 2 & 0.712 & 0.665 & -0.047 & -6.6 \\
\hline Sample 3 & 1.416 & 1.327 & -0.089 & -6.3 \\
\hline Sample 4 & 1.332 & 1.363 & 0.031 & 2.3 \\
\hline & & & Average: & -2.0 \\
\hline
\end{tabular}




\section{NMR and End Group Calculations}

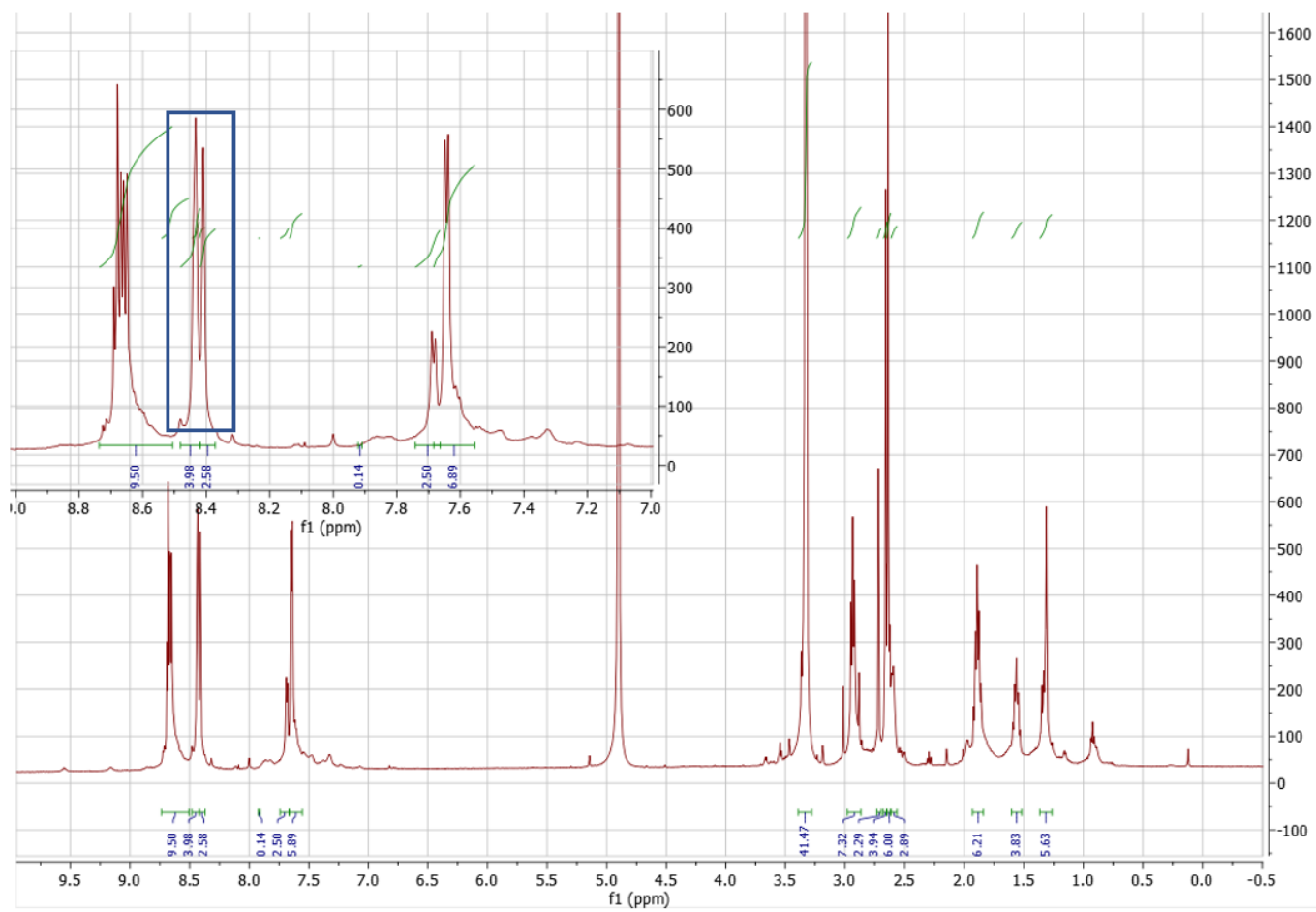

Figure S4. ${ }^{1} \mathrm{H}-\mathrm{NMR}$ Spectrum of oligomer. The inset shows comparison of end groups.

\section{Gel Permeation Chromatography}

Gel permeation chromatography was run on the ethyl-ester protected oligomeric ruthenium polypyridyl ([RuP-Et $\left.]_{\mathrm{n}}\right)$ synthetic intermediate in a chloroform system. Oligomer molecular weights and dispersities were measured using a Shimadzu pump (Shimadzu corporation), coupled with Shimadzu UV and RI detectors, controlled by an EZStart program, and calibrated with poly(styrene) standards. The column and guard column utilized were pre-packed from American Polymer Standards in chloroform (AM GPC Gel, $10 \mu \mathrm{m}$ pre-column, attached to $10 \mu \mathrm{m} 500 \AA$, and linear mixed bed columns in $\mathrm{CHCl}_{3}$; American Polymer Standards, Mentor, $\mathrm{OH}$ ) and the column temperature was maintained at $40{ }^{\circ} \mathrm{C}$. All samples were measured with a mobile phase consisting of chloroform (Sigma-Aldrich, HPLC grade, containing amylene as a stabilizer). The injection volume was $10 \mu \mathrm{L}$ and the flow rate was $1 \mathrm{~mL} \mathrm{~min}{ }^{-1}$. 


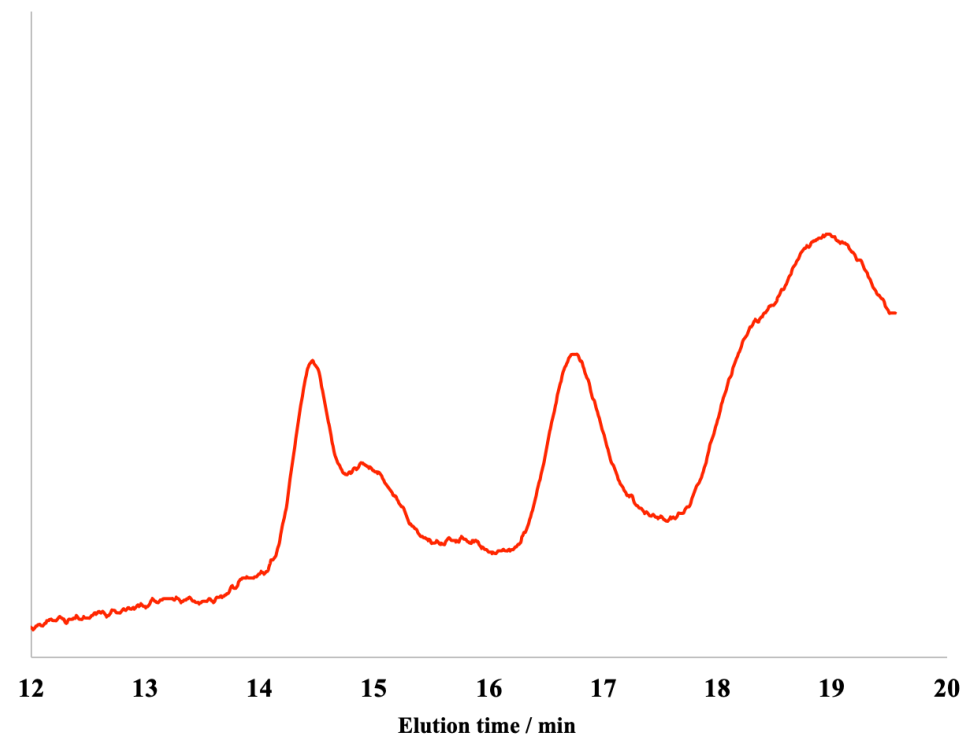

Figure S5. Gel permeation chromatography trace of oligomeric ethyl-ester precursor ([RuP-Et $\left.]_{n}\right)$

Table S2. Gel Permeation Chromatography Peak Data for ([RuP-Et $\left.]_{\mathrm{n}}\right)$

\begin{tabular}{|c|c|c|c|c|}
\hline Peak\# & $\mathrm{M}_{\mathrm{n}}$ & $\mathrm{M}_{\mathrm{w}}$ & $\begin{array}{c}\text { Dispersity } \\
\left(\mathrm{M}_{\mathrm{w}} / \mathrm{M}_{\mathrm{n}}\right)\end{array}$ & $\%$ \\
\hline Total & 3400 & 12100 & 3.48 & 100.00 \\
\hline 1 & 34400 & 35000 & 1.01 & 24.84 \\
\hline 2 & 19300 & 19450 & 1.00 & 2.68 \\
\hline 3 & 10800 & 10800 & 1.00 & 1.23 \\
\hline 4 & 5600 & 5700 & 1.01 & 41.3 \\
\hline 5 & 2300 & 2300 & 1.00 & 6.54 \\
\hline 6 & 1270 & 1300 & 1.02 & 23.41 \\
\hline
\end{tabular}

There were six distinct peaks in the GPC trace ranging from $\mathrm{M}_{n}=1270$ to $\mathrm{M}_{n}=34400$. The dispersity $\left(\bigoplus_{\mathrm{M}}\right)$ of the entire sample was 4.3 , however, if we consider only the oligomers ranging up to 10 units long and comprising the majority of the sample, the $\mathrm{Ð}=1.56$. All peaks exhibited a narrow dispersity (1.00-1.02). Each peak is likely a combination of similar chain lengths that elute from the column together, owing to columns that are packed with crosslinked poly(styrene) beads specializing in separations of up to $1,000,000$. It is likely that all chain lengths of the oligomer exist in the sample and that the distinct peaks observed are a mixture of similar lengths that elute at similar times. For example, the 7 mer peak is likely composed of chain lengths ranging from 5 to 9 units that elute at similar times. These results indicate the solution contains a broad distribution of chain lengths. 